\title{
Techniques for Obtaining Robust, Real-Time, Colour-Based Vision for Robotics
}

\author{
James Brusey and Lin Padgham ${ }^{\star}$ \\ Department of Computer Science, RMIT University, \\ P.O. Box 2476V, Melbourne 3001, Australia \\ \{brusey, linpa\}@cs.rmit.edu.au
}

\begin{abstract}
An early stage in image understanding using colour involves recognizing the colour of target objects by looking at individual pixels. However, even when, to the human eye, the colours in the image are distinct, it is a challenge for machine vision to reliably recognize the whole object from colour alone, due to variations in lighting and other environmental issues. In this paper, we investigate the use of decision trees as a basis for recognizing colour. We also investigate the use of colour space transforms as a way of eliminating variations due to lighting.
\end{abstract}

\section{Introduction}

In many domains artificial vision is the primary mechanism for a robot to sense its environment. In the domain of robot soccer it is important that vision is fast, relatively reliable in recognizing objects and is adequate for the robot to move and act appropriately in the environment. We have been exploring mechanisms to improve the reliable discrimination of objects based on colour.

In previous years we have used a vision system based on RGB (red, green and blue) pixel classification which has been manually tuned to the particular variations of colour and lighting. This year we have experimented with alternatives to RGB, such as HSL (Hue, Saturation, Luminescence) [6] and normalized RGB and evaluated the use of a decision tree learning algorithm for training the system to recognise objects from their colour in a variety of lighting conditions. This has resulted in improved object recognition.

In the following sections we describe the operating environment and the techniques explored in our efforts to improve the quality of information obtained from the vision system.

\section{General and Specific Domain Information}

Our work is focussed primarily on providing a vision system for use with an autonomous, mobile, soccer-playing robot, participating in the middle size league

\footnotetext{
* Part of this work was supported by CSIRO Mathematical and Information Sciences, Australia
} 
of the Robot Soccer World Cup (RoboCup) [8]. In this scenario two teams of $4-5$ robots ${ }^{1}$ play against each other on a green carpeted field, surrounded by white walls with a blue goal at one end and a yellow goal at the other. The soccer ball is bright orange. ${ }^{2}$ All the robots are required to be mostly black, with a light blue or purple marker to indicate team membership. Robots may be up to $80 \mathrm{~kg}$ in weight and up to $80 \mathrm{~cm}$ high. All sensing devices must be located on board the robots - i.e. it is not possible to have stationary cameras perceiving the environment and then transmitting information to the robots. However communication is allowed, and an off-board system may be used for computation if desired. Thus information perceived by one robot may be communicated to other robots.

Our robots currently have vision as their only sensor, and although we plan to add some additional sensors, vision will remain the primary mechanism for object recognition and an important mechanism for self-localization. While some level of self-localization is critical (e.g. to avoid kicking the ball towards the wrong goal), we are not aiming to achieve a high level of accuracy of location information. A human soccer player is able to play with only a rough knowledge of their own location, and so we feel that a rough knowledge is all that is required for a robotic soccer player.

Vision is also the only mechanism used for estimating distance from objects, though we may add tactile sensors for detecting when objects are very close.

The robots are quite fast-moving, with a top speed of over $2 \mathrm{~ms}^{-1}$. Information from the vision system is the primary means used to detect an impending collision. It is essential therefore that the vision system is able to process a large number of frames per second. Consequently the speed of processing for each frame of visual data is crucial. Our initial aim for the vision processing speed was 15 frames per second. This means that the robot, when at top speed, will move up to $13 \mathrm{~cm}$ before it has completely processed visual information.

Our robots are using Logitech QuickCam ${ }^{\mathrm{TM}} \mathrm{VC}$ cameras mounted in a fixed position on the robot at a height of $325 \mathrm{~mm}$ from the ground and with the camera pointing at an angle of about 17 degrees downwards from the horizontal. The field of view is 45 degrees wide and we do not currently use any distorting lenses (such as a fish eye lens). The camera has a maximum resolution of $320 \times 240$ pixels but we currently use the $160 \times 120$ mode.

The main processing phases in the vision system are:

1. (grab) get frame from camera

2. (smooth) apply smoothing

3. (classify) classify each pixel according to colour as belonging to a particular object

4. (segment) divide up image into segments of the same classification

5. (filter noise) discard small segments

6. (locate) estimate distance and angle to object associated with each segment

\footnotetext{
${ }^{1}$ There were 5 robots per team in 1997 and 1998, but only 4 per team are planned for 1999.

2 The ball is defined in the regulations as being red, but the actual colour of the ball used is described by most people as bright orange.
} 
The work we are presenting in this paper is focussed on step 3 (classification).

\section{Classification}

Classification has the aim of deciding which pixel belongs to which type of object. Each pixel is 24 bits with 8 bits each for red, green and blue. This format is known as True Colour. The red, green and blue (RGB) values for an object depend mainly on the colour of the object, but also on the colour of the lighting and level of illumination, reflections from other objects, shadows and the accuracy of the camera. For a moving robot, some of these factors will change from frame to frame. Even for a single object of uniform colour, a wide variety of different RGB values will be detected.

In order to process the image as quickly as possible, and because classification has to be performed on every pixel, it is important that this step is very simple. One approach is to compare each component of the pixel (i.e. red, green and blue) to a set of minimum and maximum thresholds. Another approach is to use a look-up table (LUT) with one element for each possible pixel value.

\subsection{Threshold Classification}

When using thresholds [11], the threshold values can either be selected manually, or by collecting pixel samples for each object type and using the mean and variance of each pixel component to determine the thresholds. Our previous system used the latter approach, and based the threshold values on the mean, plus or minus twice the standard deviation.

The thresholds form a bounding box in the pixel feature space, aligned with the axes. Every pixel colour inside the box is considered to belong to that object. There are two main problems with this approach. The first is that it assumes a distribution that fits neatly into a box, and particularly, one aligned with the axes. Second, the boxes (and thus the thresholds) sometimes overlap.

Most objects have both specular highlights and shadowed regions which, if used in finding the thresholds, tend to expand out the threshold box so that it tends to find false positives. If they are excluded from the calibration of thresholds, these parts of the object are not detected, or in other words, they will be false negatives. We found that when using the threshold approach, the trainer had to be careful to select that part of the object that was neither too dark nor too bright as the basis for training. When this approach was used, about half the object was recognized, which was typically just enough to allow the object to be tracked, as long as the lighting remained constant.

When more extensive training was performed, the thresholds tended to overlap. The previous system allowed for this by checking the thresholds in a set order. Unfortunately, the order that was used put the ball first, which tended to result in a lot of false positives for the ball. A better approach might be to adjust the thresholds to avoid overlap. 


\subsection{Look-Up Table Classification}

Another way to train a system to recognize a particular colour, is to use a look-up table (LUT) [5, 7], with one element for each possible colour $\left(2^{24}\right.$ bytes are required for a 24 bit pixel feature space). LUTs are often implemented in hardware and closely associated with the camera. Instead, we implemented the LUT in software, which had the benefit that the main memory was the only limitation on the size of the LUT, and in fact we initially used a $16 \mathrm{Mb}$ table size. (Later we dropped the least significant bit from each component, reducing the LUT size to $2 \mathrm{Mb}$.) The LUT can be manually trained by collecting pixel samples for each object. Unfortunately, this typically produces a sparsely filled array and subtle variations in lighting may prevent pixels from being classified because the training didn't include that combination of red, green and blue.

Therefore, a common augmentation of the LUT scheme is to find a generalized representation of the colour associated with a particular object and then to fill the LUT based on generalized representation. Since populating the LUT only needs to occur during training, the processing time per frame is still fast.

Given the variability of the data, what is needed is a colour generalization from actual mapped data points. It is important that this generalization be at the right level for the environment. As the RoboCup environment has very distinct colours, it should be possible to have a fairly coarse grain generalization.

A common first step in generalising colour is to transform the raw RGB (red, green and blue) components into a more appropriate colour space. The problem with RGB data is that the components are highly correlated - for example, as the lighting increases, there is a corresponding increase in all components. The correlation between red and green can be seen in Figure 1. The aim of converting to a different colour coordinate space is to reduce the correlation between the coordinates.

\subsection{Alternative Colour Spaces}

The approach used for our past RoboCup efforts involved finding fixed maximum and minimum thresholds for each of the red, green and blue (RGB) colour components based on the tristimulus model [9]. The thresholds were determined by first establishing a training set which mapped colours (RGB triplets) to object identifiers. The variance and mean for the set of colours associated with any one object where then found, and the maximum and minimum thresholds based on this.

A number of past RoboCup teams have used variants of HSL (such as HSB or HSV [3]) for colour discrimination since it separates apparent "colour" from "brightness". The brightness values of these schemes generally do not correspond well with human perception of brightness, however this is not necessarily a problem when the aim is to discriminate colours rather than to reproduce them accurately.

Even though it is useful to separate out the measure of brightness, this coefficient cannot necessarily be discarded. In the case of RoboCup, one of the objects 


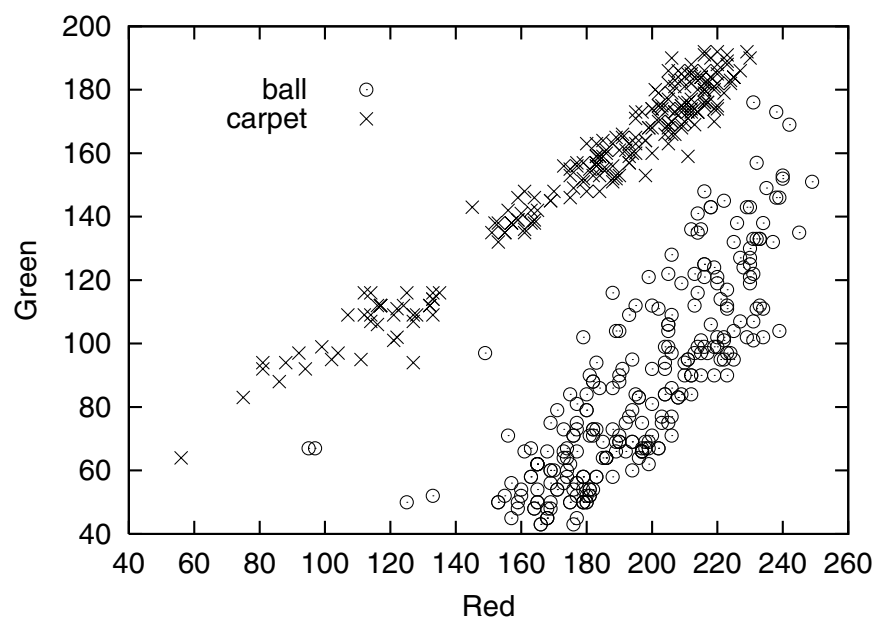

Fig. 1. Red versus Green in the RGB colour space, for sampled data for the green carpet and the orange ball.

that needs to be recognized is largely black. This object becomes impossible to differentiate from other objects if the brightness factor is ignored. To see this, we projected sampled data onto a two dimensional plane. The particular projection that we tried has previously been used with some success by Batchelor and Whelan [5] for colour discrimination of different types of apples. They refer to this projection as $\Gamma(X)$ and it is defined by:

$$
\begin{aligned}
& U=\frac{R-B}{\sqrt{2}(R+G+B)} \\
& V=\frac{2 B-R-G}{\sqrt{6}(R+G+B)}
\end{aligned}
$$

where $\Gamma(X)=(U, V)^{T}$. Unfortunately, in this colour space, samples from the black object are widely spread over much of the space and are not differentiable from the clusters of points for other objects. This yielded poor results when it was used as a basis for colour generalization (see Table 1).

One problem we found in using HSL coordinate space was that the hue component is measured as an angle around a colour circle and therefore wraps around to zero. This can result in different parts of the same object mapping to opposite parts of the hue axis, effectively splitting in two the cluster of data points for that object, as shown in Figure 2. Therefore, it is inappropriate to summarize hue using statistical mean and variance. Changing the mapping of the hue so that the split occurs at another point may solve the problem in some cases but is just as likely to move the problem elsewhere. 


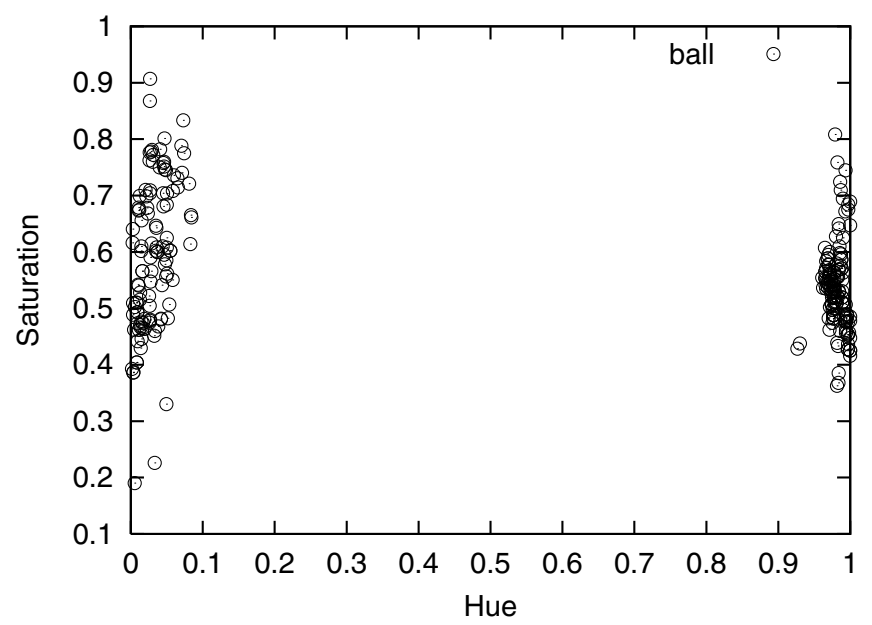

Fig. 2. Hue versus Saturation in HSL colour space, for data for the ball. Note that the data for the ball has wrapped around from 1 to 0 .

This problem is similar to that found in RGB space, where the cluster of data points for the colour of an object may be of an odd shape. Changing coordinate systems doesn't necessarily remove the unusual characteristics of the shape of the cluster.

In early results, using HSL showed clear gains in robustness of object identification and the issue of colours being wrapped around the hue axis could be dealt with using a decision tree rather than a thresholding approach (described below).

An alternative method for dealing with specular highlights and shadowed areas (which cause wide variation in RGB coordinates under different lighting conditions), is to normalize the RGB coefficients [7]:

$$
\begin{aligned}
r & =\frac{R}{R+G+B} \\
g & =\frac{G}{R+G+B} \\
b & =\frac{B}{R+G+B}
\end{aligned}
$$

Since $r+g+b=1$, one component can be dropped (usually $b$ ) as it provides no information. One of the original components, such as $G$, can then be added in to gain a measure of the brightness level. The transformed triplet is then $(r, g, G)$. We refer to this colour space informally as $r g G$.

This mechanism is quicker to compute than HSL, and it produced colour classification results which were almost as good. 
Another colour space which has been used previously to assist colour generalization [7], is HSI. HSI can be calculated from RGB as follows:

$$
\begin{array}{r}
I=\frac{R+G+B}{3} \\
S=\frac{1-\min (R, G, B)}{R+G+B} \\
H=\arctan \left(\frac{3(G-B)}{2 R-G-B}\right)
\end{array}
$$

\subsection{Using Decision Trees for Learning Colour}

Whichever colour coordinates are used (RGB, HSL, rgG or HSI), there is a tendency, especially where the training data is extensive, for the resulting thresholds for different objects to overlap. Thus, although thresholding is fast it can often lead to conflicts in object identification.

An alternative approach is to use a decision tree structure which allows for a more detailed partitioning of the feature space than that allowed by thresholds. The added degrees of freedom give an effect analogous to having multiple threshold cubes associated with any particular object.

If appropriate rules are known, decision trees can be designed manually. More often they are created from training data, using a learning algorithm. We used the C4.5 learning algorithm [10], which is an industrial strength, decision tree based, machine learning algorithm. It is a batch mode learning algorithm, which means that it prepares the decision tree on the basis of all the training examples, rather than adaptively changing the tree as it receives more data.

Decision trees, when based on continuous values have the advantage that they can always classify any data, unlike thresholding which may leave some data unclassified. Also no data is multiply classified as can occur with thresholding.

Figure 3 shows an example of a pruned decision tree. Pruning is performed automatically by the $\mathrm{C} 4.5$ algorithm, and is done by removing parts of the tree which do not significantly affect the error rate. The leaf nodes show two numbers $(N / E)$ after the classification. The first $(N)$ is the number of training cases which ended up at that leaf node. The second $(E)$ is the predicted error rate for that node if $N$ unseen cases were classified by the tree.

\subsection{Classification Results}

The results for the threshold and decision tree classifiers are shown in Table 1. The overall result is that the decision tree classifier is generally better than the threshold classifier and generally classifies $97 \%$ of data correctly. The HSL and HSI colour spaces wrap around and so they were inappropriate for use with the threshold classifier. The RGB colour space defeated our expectations and gave the best results. There is some margin for error in these results, but we were still surprised to see the RGB colour space rate so well.

The result for the $\Gamma(X)$ space shows that, for this type of data, three dimensional colour data is required to discriminate between the different classes. 


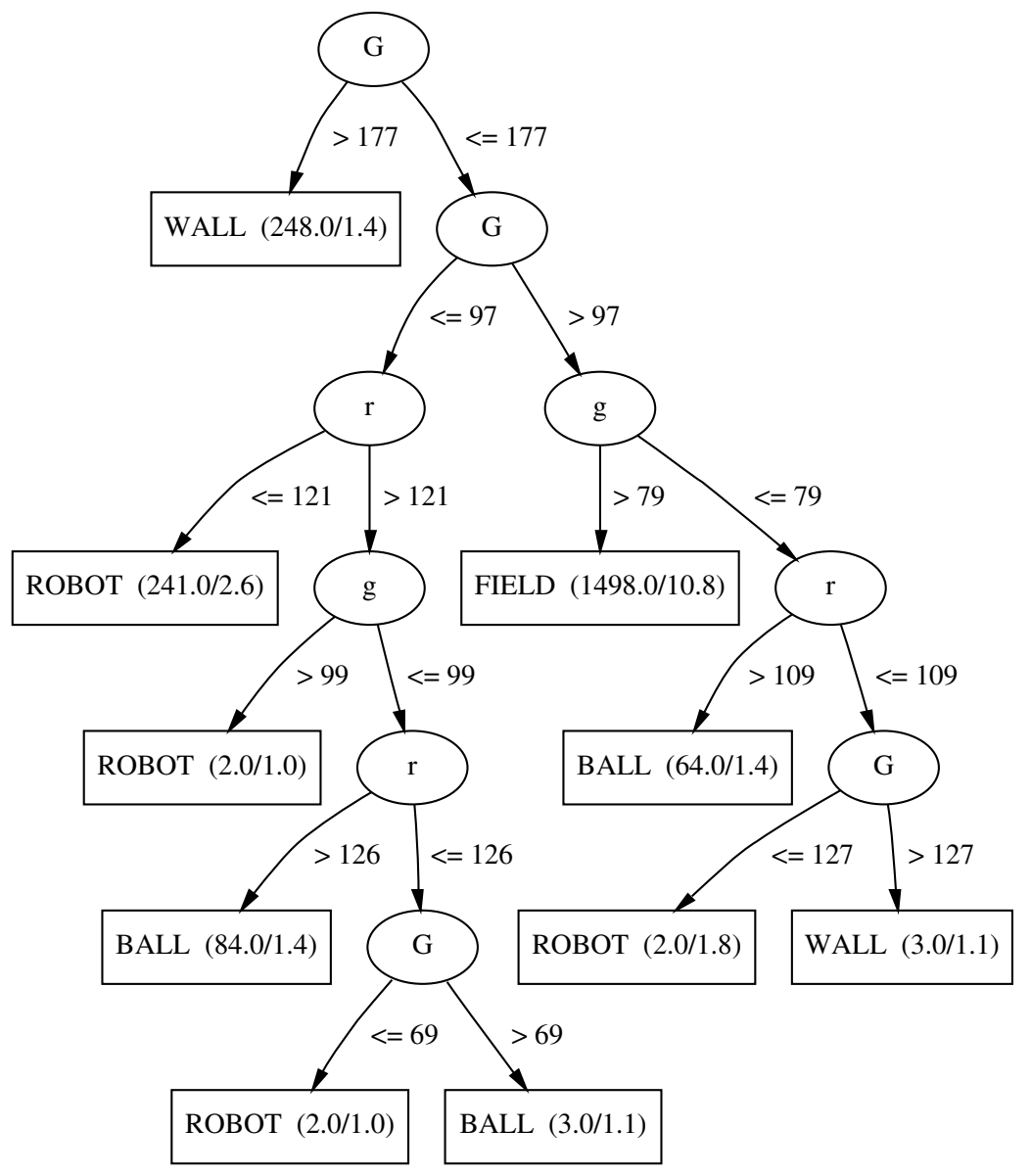

Fig. 3. An example decision tree. The colour model used in this case was rgG, where the " $\mathrm{G}$ " component roughly corresponds to brightness. All components are scaled in the range $[0,255]$. 
Table 1. Summary of results for different colour models. All results were based on tests on data not seen during training (using a 50/50 split between training and test data).

Threshold classifier

Colour Correct Incorrect Multiply Unclassified

\begin{tabular}{ccccc} 
model & \multicolumn{3}{c}{ classified } \\
\hline rgG & $80.0 \%$ & $1.4 \%$ & $8.8 \%$ & $9.8 \%$ \\
RGB & $84.9 \%$ & $2.1 \%$ & $6.0 \%$ & $7.0 \%$
\end{tabular}

Decision tree classifier

\begin{tabular}{ccccc}
\hline$\Gamma(X)$ & $87.5 \%$ & $12.5 \%$ & - & - \\
HSI & $97.5 \%$ & $2.5 \%$ & - & - \\
HSL & $97.6 \%$ & $2.4 \%$ & - & - \\
rgG & $97.3 \%$ & $2.7 \%$ & - & - \\
RGB & $97.9 \%$ & $2.1 \%$ & - & - \\
\hline
\end{tabular}

\section{Related work}

Several other papers presented at the workshop dealt with the issue of colour recognition [1, 2, 4, 12]. Akita [1] focussed on the development of specialised hardware. RGB data supplied by the hardware is transformed to HSV by a look-up table before thresholds for each of the $\mathrm{H}, \mathrm{S}$, and $\mathrm{V}$ components are used to distinguish the colours of different objects. This classification technique appears to be the same as that described in Section 3.1.

Amoroso et al. [2] found that with the HSI transform, the different objects on the field could be discriminated using the hue component alone. Discrimination is trained by examining the hue histogram for valleys. A feed-forward neural network is used to learn the correspondence between hue "modes", extracted from the histogram, and actual objects. They also deal with the problem of having separate hue regions that correspond to a single object (as discussed in Section 3.3).

Use of hue alone may not allow for recognition of black objects, which we found hard to discriminate unless brightness was taken into account (Section 3.3). Their results show a smaller percentage of misclassifications than we were able to achieve but this may be related to our attempts at recognising black objects.

Bandlow et al. [4] start with data in the YUV colour space. A classification look-up table is trained for the UV components. For the Y component, which corresponds to the pixel brightness, minimum and maximum thresholds are determined for each object.

Terada et al. [12] mention that they use Mehalanobis distance measures to classify colours and train with samples of each colour.

One difficulty with comparing results is that the quality of the source data is subject to error that is critically dependent on the type of camera. We found 
that it was quite difficult to tune the QuickCam VC so that the colours looked realistic. At the boundaries between light and dark objects, lines of bright red sometimes appeared due to the way the colours are detected using a mask.

\section{Evaluation and Discussion}

Our goal in working with the vision system was to develop a sound perceptual footing for a cognitive system that approaches human-like behaviour within this domain. Our longer term goal is to explore issues in building, maintaining and reasoning with approximate world models in a dynamic real-time domain. Our main requirements for the vision system were therefore:

1. to achieve robust object identification (this implies virtually no false positives or negatives); when an object is present in the visual field it should be seen and recognized, the system should not "see" anything that is not actually in the visual field;

2. to process frames at a rate sufficient to judge direction of movement of target objects with some rough idea of speed;

3. to coordinate vision with movement to avoid problems such as hitting objects before it has registered that they are there, or not finding the ball due to having moved past it by the time the robot has processed that it was "seen".

The use of a decision tree colour classifier has addressed problems due to oddly shaped colour clusters, while the learning of this decision tree has ensured the appropriate amount of generalization/specialization for the environment as well as removing much of the tediousness previously associated with establishing appropriate threshold values. These combined measures have resulted in robust object identification and we appear to have eliminated problems with both false negatives and positives.

We are currently getting a frame rate of 16 frames per second, which we believe will be sufficient for judging direction of movement and for coordinating with the robot's own movement.

Our vision system does not address vision issues associated with such things as reading text or recognizing specific shapes. Such things are certain to be useful for self-localization if incorporated into the RoboCup environment as is being discussed. However, based on human behaviour it does not seem that such abilities should be necessary for this domain. We are interested rather in exploring other approaches to the issue of adequate self-localization in the absence of detailed, accurate positioning data obtained either from visual nuances or other percepts.

Two aspects of vision that we expect to address in our continued work are the use of a movable camera, and if possible, obtaining of a wider angle of vision. Both these directions aim to address the problem of seeing too little at any one time. For all players, but particularly for the goalie, it is important to have a reasonably wide visual coverage. 
We are hopeful that the improvements made to date will give us adequate perceptual information to make use of our action selection and coordination mechanisms, as well as allowing us to explore dynamic teamwork.

\section{References}

[1] Junichi Akita. Real-time color detection system using custom LSI for high-speed machine vision. In Veloso [13].

[2] Carmelo Amoroso, Antonio Chella, Vito Morreale, and Pietro Storniolo. A segmentation system for soccer robot based on neural networks. In Veloso [13].

[3] Minoru Asada, editor. RoboCup-98 : Proceedings of the second RoboCup Workshop, July 1998.

[4] Thorsten Bandlow, Michael Klupsch, Robert Hanek, and Thorsten Schmitt. Fast image segmentation, object recognition and localization in a Robocup scenario. In Veloso [13].

[5] B. G. Batchelor and P. F. Whelan. Real-time colour recognition in symbolic programming for machine vision systems. Machine Vision and Applications, $8(6): 385-398,1995$.

[6] A. Glassner, editor. Graphics Gems, pages 448-452. Academic Press, 1990.

[7] P. Jasiobedzki, B. Down, and V. Wu. Active object detection using colour. In C. Archibald and P. Kwok, editors, Research in Computer and Robot Vision, pages 37-53. World Scientific, 1995.

[8] I. Noda, S. Suzuki, H. Matsubara, M. Asada, and H. Kitano. Robocup-97: The first robot world cup soccer games and conferences. AI Magazine, 19(3):49-59, 1998.

[9] W. K. Pratt. Digital Image Processing. John Wiley and Sons, 1991.

[10] J. R. Quinlan. C4.5 : programs for machine learning. Morgan Kaufmann, 1993.

[11] M. Sonka, V. Hlavac, and R. Boyle. Image Processing, Analysis and Machine Vision. Chapman and Hall, 1993.

[12] K. Terada, K. Mochizuki, A. Ueno, H. Takeda, T. Nishida, T. Nakamura, and A. Ebina. A method for localization by integration of imprecise vision and a field model. In Veloso [13].

[13] Manuela Veloso, editor. Robocup-99 : Proceedings of the Third RoboCup Workshop, July 1999. 